\title{
Chinesische Li-Bai-Vertonungen in Jahren der Unruhe
}

\section{Chinese Li Bai Settings in Years of Upheaval}

This essay explores the way in which texts by the eighth-century poet Li Bai served as a basis for twentieth-century Chinese composers to reconstruct their auditory identities and redefine Chinese musical styles. Focussing on art songs written during the Second Sino-Japanese War of 1937-1945, this essay offers insights into the ways that Li Bai's texts were used and abused as a cultural reference by composers in the Nationalist, Communist and Japanese-occupied territories of wartime China. A brief analysis of three songs by Xian Xinghai and Jiang Wenye illustrates these composers' struggle to define their national and cultural identities within the form of art songs. Notes on the performance of such songs organised by Chinese client regimes in the Japanese-occupied territories reveal the highly politicised nature of Li Bai's texts during this time of military conflict and socio-economic upheaval. This essay includes the first-ever survey of $20^{\text {th }}$-century Chinese composers' art songs based on Li Bai's texts, and furthers our understanding of these songs as a result of complex transcultural processes.

Gedichte Li Bais gehören wohl zu den meistvertonten im chinesischsprachigen Raum. Dies mag auch an Li Bais außergewöhnlichem Stil liegen - so wird er in einem Standardwerk zur chinesischen Literaturgeschichte folgendermaßen beschrieben: »Li Po ist der musikalischste, vielseitigste und fesselndste Dichter Chinas, ein Mozart der Wörter. « ${ }^{1}$ Zweifelsohne hat Li Bai auf seinen Reisen verschiedenste sprachliche und musikalische Traditionen kennengelernt, die Eingang in sein Schreiben gefunden haben. Viele seiner Gedichte gebrauchen lyrische Formen, die den musikalischen Vortrag antizipieren, oder sind feuchtfröhlichen Ritualen gewidmet, die den Rahmen für entsprechende Gesänge bieten könnten. Vor dem zwanzigsten Jahrhundert wurden Li Bais Gedichte insbesondere zum Vortrag mit der Qin 琴 vertont, dem Instrument der Literaten. ${ }^{2}$ Dieser Essay widmet sich jedoch den Li-Bai-Vertonungen chinesischer Komponist ${ }^{*}$ innen des zwanzigsten Jahrhunderts,

DOI: $10.26045 /$ po-010

1 "Li Po is the most musical, most versatile, and most engaging of Chinese poets, a Mozart of words. « Paul W. Kroll: Poetry of the T'ang Dynasty, in: The Columbia History of Chinese Literature, hg. von Victor H. Mair, New York: Columbia University Press, 2001, S. 274-313, hier S. 296. Alle Übersetzungen ins Deutsche, so nicht anders angegeben, sind von der Autorin.

2 Yang Sihui: Li Bai shici gequ yanjiu, in: Journal of Anhui University of Technology 32/6 (2015), S. 59-62. 
und zwar solchen, die Kunstlieder im engeren Sinne darstellen, insbesondere jenen aus den 1930er und frühen 1940er Jahren, als auch Harry Partch Li Bais Texte vertonte. Er endet mit einem Ausblick über diese Jahre der Unruhe hinaus.

Wie sich die politische Unsicherheit und militärische Unruhen in der ersten Hälfte des zwanzigsten Jahrhunderts auf das kulturelle Schaffen in China auswirkten, ist vielfach untersucht worden. ${ }^{3}$ Die zunehmende koloniale und missionarische Präsenz in den Küstenregionen Chinas gegen Ende des neunzehnten Jahrhunderts infolge der Opiumkriege und chinesische Auslandsstudenten, die direkt oder über Japan europäische Musikpraktiken nach China brachten, führten zu immensen Veränderungen im Musikverständnis und musikalischen Leben Chinas. Komponist ${ }^{*}$ innen begannen sich als solche zu verstehen, und Konservatorien bildeten Musiker ${ }^{\star}$ innen nach europäischem Vorbild aus. ${ }^{4}$ Europäische Notationssysteme wie die Chevésche Ziffernnotation und das heute gebräuchliche ১fünf-Linien-Notensystem`wurden adaptiert. Normen zur äquidistanten Stimmung, die schon im späten 16. Jahrhundert errechnet worden waren, ${ }^{5}$ wurden vielfach diskutiert und mit der Verbreitung europäischer Tasteninstrumente ab Mitte des neunzehnten Jahrhunderts als Standard akzeptiert. ${ }^{6}$ Da der edukative Nutzen von Liedern als erwiesen und dem Wachsen von Nationalbewusstsein zuträglich galt, ${ }^{7}$ entstand eine große Zahl sogenannter Schullieder. Dabei wurden zunächst häufig schon bekannte Melodien - wie etwa Frère Jacques - zu chinesischen Texten gesetzt. Später wurden neue Melodien zu Texten mit oft stark politisierendem, nationalistischem Charakter komponiert. Die zunehmende Bedrohung durch Japan, die letztlich in den Zweiten Sino-Japanischen Krieg und die japanische Besetzung chinesischer Küstengebiete mündete, gab Anlass zu intensivierter kompositorischer Tätigkeit - insbesondere

So sind die Auswirkungen der japanischen Besetzung während des Zweiten Sino-Japanischen Krieges Gegenstand der Forschung in Literatur- und Filmgeschichte. Vgl. hierzu Edward Gunn: Unwelcome Muse. Chinese Literature in Shanghai and Peking, 1937-1945, New York: Columbia University Press, 1980; Poshek Fu: Passivity, Resistance, and Collaboration. Intellectual Choices in Occupied Shanghai, 1937-1945, Stanford, CA: Stanford University Press, 1993; ders.: The Ambiguity of Entertainment. Chinese Cinema in Japanese-Occupied Shanghai, 1941 to 1945, in: Cinema Journal 37/1 (1997), S. 66-84.

4 Eine umfassende Geschichte der Entwicklung neuer chinesischer Musik im frühen zwanzigsten Jahrhundert findet sich in Liu Ching-Chih: A Critical History of New Music in China, übers. von Caroline Mason, Hong Kong: Chinese University Press, 2010, S. 23-286.

5 Joyce Lindorff: Missionaries, Keyboards and Musical Exchange in the Ming and Qing Courts, in: Early Music 32/3 (2004), S. 403-414, hier S. 413.

6 So stellte sich die Frage nach mikrotonalem Komponieren der nach europäischem Vorbild ausgebildeten und auf das >Aufholen europäischer Tradition ausgerichteten musikalischen Elite zu diesem Zeitpunkt nicht.

7 Zum engen Verhältnis zwischen Musik und Politik im modernen China siehe Barbara Mittler: Dangerous Tunes. The Politics of Chinese Music in Hong Kong, Taiwan, and the People's Republic of China since 1949, Wiesbaden: Harrassowitz, 1997, S. 36-125. Eine kurze Einführung in die frühe chinesische Musikkosmologie und die Symbolkraft und Bedeutung von Musik in der Politik des frühen China findet sich in Lothar von Falkenhausen: Suspended Music. Chime-Bells in the Culture of Bronze Age China, Berkeley: University of California Press, 1993, S. 310-324. 
im Bereich solcher Lieder. ${ }^{8}$ Glaubt man dem historiografischen Standardnarrativ, so wurden Komponist ${ }^{\star}$ innen und Musizierende zu politischen Aktivisten, ihre propagandistischen Lieder zu Waffen, die dem Volk, vereint im erbitterten Widerstandskampf, eine Stimme verliehen. ${ }^{9}$ Unruhe stifteten aber auch die Konflikte zwischen der Nationalen Volkspartei und der Kommunistischen Partei Chinas, die nach dem Kriegs-Interludium zu einem offenen Bürgerkrieg führten. Auch in diesem Konflikt wird Musiker`innen eine entscheidende politisierende Rolle zugeschrieben.

Inmitten von Bombenalarm und Gewehrfeuer - wo blieb da noch Muße für Kunstlieder, geschweige denn für Lieder mit uralten, gefühlsbetonten Texten? Allen Widrigkeiten zum Trotz waren die späten 1930er und 1940er Jahre, die häufig als Übergangszeit zwischen einer politisch instabilen, aber kulturell florierenden Republik und der `Befreiung « durch die KPCh angesehen werden, eine entscheidende Phase der Entwicklung neuer Musik in China: ${ }^{10}$ eine Zeit, in der chinesische Kom-

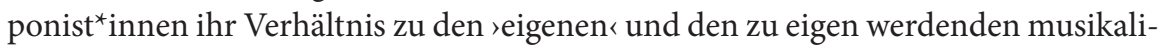
schen Traditionen zu definieren und zu festigen begannen. In der chinesischen Literatur- und Kunstgeschichte gilt der Rückgriff auf traditionelle Formen während des Kriegs als Ausdruck des Wunsches der Schriftsteller und Künstler nach Ordnung in den besetzten und umkämpften Gebieten. ${ }^{11}$ Und auch Musiker ${ }^{*}$ innen fanden auf der Suche nach Ruhe Zugang zu alten Texten und alten literarischen und musikalischen Formen. ${ }^{12}$ Abseits vom Alltagstrubel wurden Klaviersonaten und Kunstlieder komponiert. ${ }^{13} \mathrm{Li}$ Bais Texte scheinen bei der Suche nach passenden Ausdrucksformen einen Anlaufpunkt dargestellt zu haben.

Als Standort von Chinas erstem Konservatorium wurde Shanghai ab 1927 Zentrum der Entwicklung neuer Musik. Die in Shanghai ansässigen Komponist*innen vertonten die Texte Li Bais in ersten Kunstliedern, so zum Beispiel Nach Jiangling (Xia Jiangling 下江陵), vertont von Huang Zi 黃自 (1904-1938), einem der Begründer der neuen Musik in China. Auch sein Schüler He Lüting 和綠汀 (1903-1999),

8 Joshua Howard: »Music for a National Defense«. Making Martial Music during the AntiJapanese War, in: Cross-Currents 4/1 (2015), S. 238-284, hier S. 277.

9 Dieses Narrativ wird auch weiterhin bedient, vgl. Tang Xiaobing: Radio, Sound Cinema, and Community Singing. The Making of a New Sonic Culture in Modern China, in: TwentiethCentury China 45/1 (2020), S. 3-24.

10 Ich verwende `neue Musik « im engeren Sinne für Musik, die unter europäischem Einfluss von chinesischen Komponist ${ }^{*}$ innen geschrieben wurde, die Teil der in Konservatorien, Universitäten und Filmproduktionsfirmen institutionalisierten Elite waren.

11 Gunn: Unwelcome Muse, S. 268 und 110; Carolyn FitzGerald: Fragmenting Modernisms. Chinese Wartime Literature, Art, and Film, 1937-49, Leiden/Boston: Brill, 2013, S. 10-12.

12 Die Konfuzianische Tempelmusik (Kōbyō taisei gakushō/ Kongmiao dacheng yuezhang 孔廟大 晟樂章) von Jiang Wenye zum Beispiel ist ein Versuch der musikalischen Rekonstruktion konfuzianischer Rituale, vgl. David Der-Wei Wang: The Lyrical in Epic Time. The Music and Poetry of Jiang Wenye, in: The Lyrical in Epic Time. Modern Chinese Intellectuals and Artists Through the 1949 Crisis, New York: Columbia University Press, 2015, S. 193-235, hier S. 207-211.

13 Ma Sicong und Jiang Wenye gelten als Pioniere der Klaviersonate in China, sie schrieben auch eine große Zahl an Kunstliedern (vgl. Liu: A Critical History, S. 316f.). 
dessen Kunstlieder zu den meistaufgeführten dieser Zeit zählen, wandte sich immer wieder Gedichten Li Bais zu. So vertonte er in den 1930er Jahren Erinnerung an die Schönheit von Qin (Yi Qin'e 憶秦娥), Pusaman 菩薩蠻 und Gedanken in stiller Nacht (Jing ye si 靜夜思). Letzteres wurde auch von der einzigen Komponistin in dieser illustren Runde, Xiao Shuxian 蕭淑嫺 (1905-1991), vertont. ${ }^{14} 1933$ vertonte Liu Xue’an 劉雪庵 (1905-1985) Einer Flöte lauschen in Luoyangs Frühlingsnacht (Chunye Luo cheng wen di 春夜洛城聞笛). Und sogar Xian Xinghai 洗星海 (19051945), dank seiner revolutionären und anti-japanischen Lieder und der Kantate vom Gelben Fluss (Huanghe da hechang 黃河大合唱) der vielleicht bekannteste chinesische Komponist dieser Generation außerhalb von China, vertonte Li Bais Texte: 1940, während seines Aufenthaltes in Xi'an unweit der Hochburg der Kommunistischen Partei, entstand Erinnerung an die Schönheit von Qin. ${ }^{15}$ Xian Xinghai verbrachte diese Zeit getrennt von seiner Frau, was die Textauswahl begründen könnte. ${ }^{16}$

Wie die unten besprochenen Li-Bai-Vertonungen von Doming Lam ist auch Xian Xinghais Lied für Stimme, Klavier und Querflöte gesetzt. Die Flöte, die im Gedicht schon mit der ersten Silbe als Weckruf in Erscheinung tritt, wird von einer Zusatzstimme zur Hauptattraktion des Liedes befördert: Verziert-exklamative Phrasen der Flöte werden von Arpeggien im Klavier begleitet. Die erst in Takt 13 einsetzende Stimme wirkt fast wie ein versonnen-nebensächlicher Kommentar zu der von Flöte und Klavier ohne Unterbrechung gestalteten Szenerie. Das Klavier ist dabei nicht reines Begleitinstrument, sondern steht im Dialog mit Flöte und Gesang, führt deren Melodien fort und verarbeitet die erklungenen Motive.

Wie die meisten in Europa ausgebildeten chinesischen Komponist ${ }^{\star}$ innen seiner Generation bemühte sich Xian Xinghai, seiner europäischen musikalischen >Muttersprache` Elemente der traditionellen Musik seiner geografischen Heimat beizufügen und so chinesische Identität in der ihm zu eigen gewordenen Ausdrucksform zu markieren. Dabei wurde die Annäherung an die vermeintlich reigene Tradition oftmals zu einem Rekonstruktionsprozess, der unser landläufiges Verständnis von kultureller Zugehörigkeit des Komponisten auf den Kopf stellt. Die Historizität und Authentizität der im Verlauf dieses Prozesses als chinesisch identifizierten Elemente ist dabei nachrangig (wenn auch nicht vollkommen ohne Basis in musikalischer Praxis, deren Vielfalt einer so limitierten Definition `chinesischer Elemente jedoch spottet). Die von Xian Xinghai verwendeten musikalischen Mittel galten dennoch

14 Peng Li 彭丽. Juan wei wang yue kong chang tan. Xi Ma Sicong de yishu gequ »Li Bai shi liu shou«, in: Lu Xun Yiyuan魯迅藝苑 4 (2006), S. 57-62.

15 Xian Xinghai quanji bian weiyuanhui: Xian Xinghai quanji, Band 2, Guangzhou: Guangdong Higher Education Press, 1990, S. 328-331. Liu Ching-Chih datiert das Lied auf 1939 (Liu: Critical History, S. 214), andere verorten die Entstehung nach Xi'an im Jahr 1940: Liu Qinqin: Shangbie chu, yin jianjue - Xian Xinghai yishu gequ »Yi Qin'e« fenxi, in: Journal of Xinghai Conservatory of Music 3 (2011), S. 80-85, hier S. 83.

Ebd. 


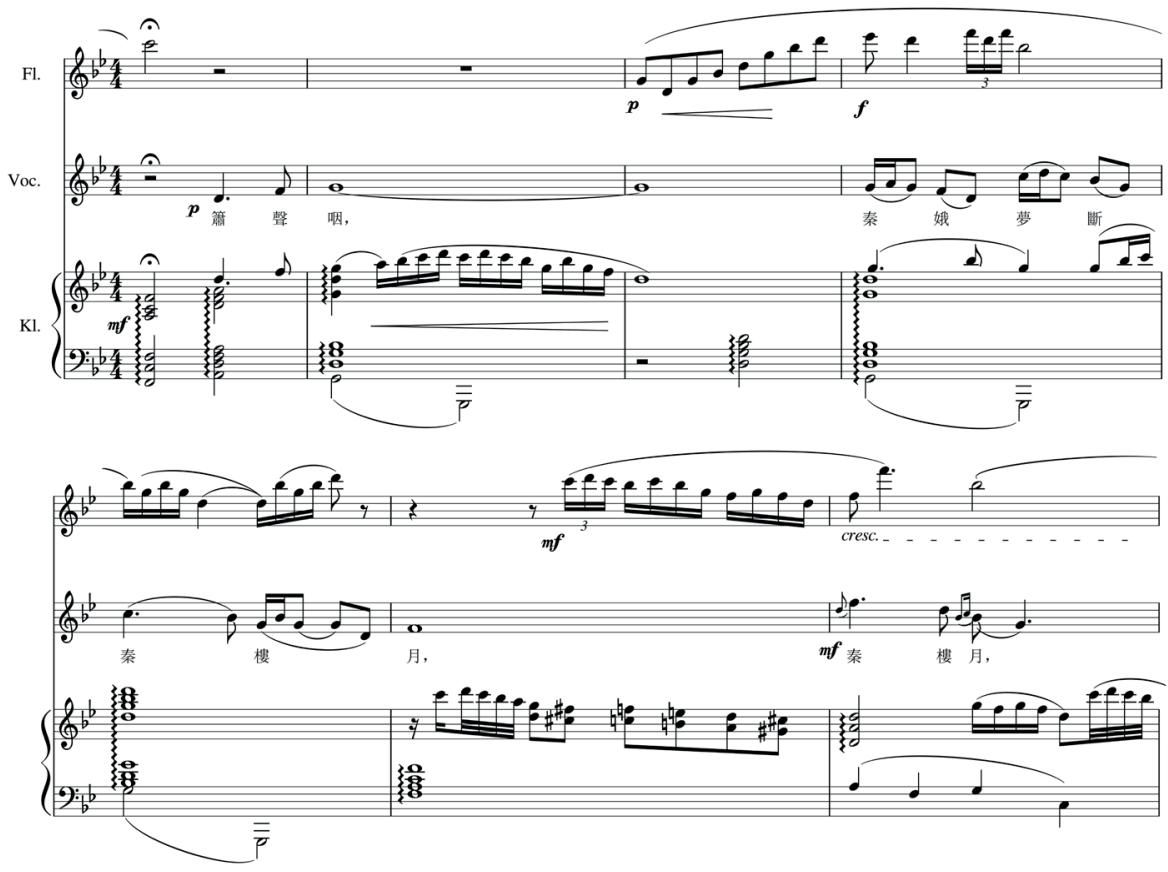

Abb. 1: Xian Xinghai: Erinnerung an die Schönheit von Qin, erster Einsatz der Gesangsstimme, Takt 13-19

- und gelten teilweise bis heute, nicht zuletzt aufgrund Xians Prominenz - als identitätsstiftend.

So definieren verschiedene musikalische Elemente Erinnerung an die Schönheit von Qin als entschieden chinesisch: Es steht in pentatonischem $y u$-Modus auf $g^{17}$ und kehrt immer wieder dahin zurück, begibt sich aber zwischenzeitlich auch in andere modale Gefilde, die jedoch keine chromatischen Abweichungen von der vorhandenen Skala verlangen, darunter gong- und zhi-Modus auf $f$. Einzige Ausnahme sind zwei chromatische Abwärtsbewegungen im Klavier, die auf die jeweiligen Höhepunkte der Verse hinführen. Alte chinesische Gesangstechniken werden durch triolische Verzierungen, Vorschläge und langsames Tremolo (Tonwiederholungen ohne neue Textsilbe) nachempfunden. Volkstümlich mutet auch die Rhythmisierung an: Schnelle Bewegungen werden oft ohne Auftakt zum Taktanfang gebracht

17 Ähnlich den europäischen Kirchentonarten ergeben sich - aufbauend auf dem in der traditionellen chinesischen Musik innerhalb einer Oktave verfügbaren Tonvorrat der zwölf sogenannten $l u ̈$ 律 (die in etwa der chromatischen Tonleiter entsprechen) - fünf pentatonische Modi: gong 宮, shang 商, jue 角, $z h i$ 徵 und $y u$ 羽. Diese weisen jeweils unterschiedliche Intervallstrukturen auf und können auf einem beliebigen Grundton der zwölf lü aufgebaut werden. So entspricht der gong-Modus auf $c$ in etwa der Skala $c-d-e-g-a$, der shang-Modus der Skala $d$-e$g-a-c$ und so fort. Für weiterführende Informationen zu diesem Thema vgl. Falkenhausen: Suspended Music, S. 280-309. 
und Endsilben über Taktstriche hinweg gehalten. So beginnen die Verse häufig mit einem punktierten Achtel plus Sechzehntel, direkt gefolgt von einer Achteltriole, oder aber mit zwei Sechzehnteln gefolgt von drei Achteln (vgl. Notenbeispiel 1). Dabei handelt es sich häufig um mit Wechselnoten auskomponierte Verzierungen. Außergewöhnlich sind auch die vielen Momente, in denen Flöte und Stimme sich in Oktaven treffen und sich leicht versetzt oder für kurze Zeit gänzlich parallel bewegen - womöglich den parallelen Bewegungen eines traditionellen Ensembles nachempfunden. Der Anfang des zweiten Teils ist belebter, Stimme und Flöte wechseln sich in schnellen Aufwärtsbewegungen ab, bevor beide zum wiegenden, gefühlvollen Ausgangsgestus zurückkehren. Wichtige Textabschnitte werden durch Wiederholungen weniger Silben musikalisch ausgekostet. Xian Xinghais Kunstlied illustriert nicht nur die Sehnsucht nach alten Formen und Klängen, sondern auch, wie selbstbewusst chinesische Komponist ${ }^{\star}$ innen zu Beginn der 1940er Jahre chinesische Elemente in für sie neue Formen integriert haben.

Wenn wir das oben beschriebene Lied mit den vielen Propagandaliedern Xian Xinghais vergleichen, wird auch deutlich, wie rigide sich letztere doch an europäische und russische Vorbilder halten. In Anbetracht des dringenden Verlangens nach Nationalbewusstsein mag dies kontraintuitiv erscheinen. Doch die Idee der Definition einer primär an den Nationalstaat - nicht etwa eine gemeinsame Schriftsprache oder andere kulturelle Praktiken - gebundenen kulturellen Identität war vergleichsweise neu. Es könnte also sein, dass Propagandalieder den Klang eines stereotypen Nationalstaates widerspiegeln, während Experimente klanglicher Identitätsfindung abseits der direkten Konfrontation mit europäischen Idealen von Staatlichkeit, also nicht in nationalistischen Kampfliedern, sondern viel eher in Kunstliedern wie jenen mit Texten Li Bais unternommen wurden.

Nicht nur in den Gebieten der Nationalisten und Kommunisten wurden Li Bais Texte vertont. Auch in den ab 1937 japanisch besetzten Regionen griff man auf seine Gedichte zurück. Jiang Wenye 江文也 (1910-1983), geboren in der damaligen japanischen Kolonie Taiwan und aufgewachsen in Japan, zog 1938 in das japanisch besetzte Peking. Als Kind des Kolonialismus fiel es Jiang schwer, Zugehörigkeiten zu finden: Er bewunderte in anderen Gebieten Chinas unbekannte und vergleichsweise moderne europäische Musik (Jiang kannte Bartók, Stravinskij und Honegger) und fand wenig Gleichgesinnte im besetzten Peking. Er war zudem von Diskriminierungserfahrungen als `Chinese in Japan geprägt. Im besetzten China hingegen genoss er das Privileg der japanischen Staatsbürgerschaft (wenn auch zweiter Klasse). In Peking wollte er nun >seine` musikalischen Wurzeln erforschen, sich von traditioneller chinesischer Musik inspirieren lassen und damit zur kulturellen Erneuerung Chinas beitragen. So erforschte er zum Beispiel konfuzianische Tempelmusik und sammelte Volkslieder. Zudem arbeitete er sich als Liederkomponist systematisch durch das Korpus chinesischer Lyrik. Seine Liederzyklen, zumeist einer Dynastie oder einem spezifischen poetischen Genre gewidmet, wirken wie Übungen nicht im Sprach-, sondern im musikalischen Traditionserwerb. Dazu gehören drei Vertonungen von Gedichten Li Bais, die in Jiangs Zyklen zu fünf- beziehungsweise siebensil- 


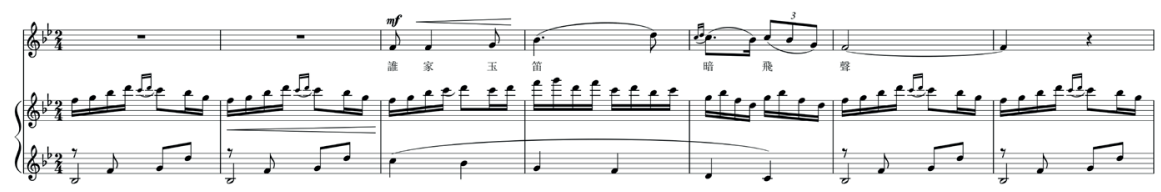

Abb. 2: Jiang Wenye: Einer Flöte lauschen in Luoyangs Frühlingsnacht, Takt 6-12

bigen Kurzgedichten von 1939 enthalten sind: Gedanken in stiller Nacht, Pagode des gelben Kranichs (Huanghe lou 黃鶴樓) und Einer Flöte lauschen in Luoyangs Frühlingsnacht. Zwischen 1944 und 1946 vertonte Jiang weitere neun Gedichte Li Bais. ${ }^{18}$

Jiangs Lieder sind bestechend einfach. Pagode des gelben Kranichs steht im gong-Modus auf $g$. Auffallend ist das Ostinato im Bass, der auftaktig die None $G-A$ vorgibt. Diese Dissonanz bleibt das komplette Lied über erhalten und wird auch gegen Ende nicht aufgelöst. Auch die weiteren musikalischen Elemente im Klavier sind sparsam: Da ist zunächst eine zweitaktige Melodie, die simultan in drei Oktaven vorgestellt und zweimal wiederholt wird, dann ein Akkord, ebenfalls über drei Oktaven, bestehend aus der Quart-Quintschichtung $d-g-a$, der wechselweise um einen Halbton versetzt erklingt, bevor zum Ausgangsakkord zurückgekehrt und dieser wiederholt wird. Die Gesangsstimme übernimmt für die erste Hälfte des Gedichtes die vom Klavier vorgegebene Melodie - mit einer Endfloskel versehen. Auch das Abweichen der Stimme von der Melodie ist simpel gehalten und nur von sehr kurzer Dauer. Gegen Ende wird die erste Melodie bis zum Verschwinden verkürzt und verhallt so mit dem Ostinato.

Einer Flöte lauschen in Luoyangs Frühlingsnacht ist ebenso einfach gehalten: Das erste vom Klavier als unbegleitete Melodie in der rechten Hand vorgestellte Motiv imitiert die dem Gedicht den Titel verleihende Flöte und wird im Folgenden als Begleitung der Gesangsstimme wiederholt (vgl. Notenbeispiel 2). Wie bei Xian Xinghai wird auch in diesem Stück mit tonaler Ambiguität gespielt - hier zwischen $z$ hi-Modus auf $f$ und gong-Modus auf $b$-, die keine Auflösung erfährt. In der viertaktigen Melodie der Gesangsstimme erkennen wir Gesten wieder, derer sich schon Xian Xinghai bediente: die Betonung des Taktanfangs - hier durch eine Synkope - sowie triolische Auftakte zu einem langgestreckten Phrasenendton. Jiang identifizierte infolge seiner Beschäftigung mit konfuzianischer Tempelmusik die Einfachheit als musikalisches Mittel und wagte es, dieses einzusetzen, entgegen dem Impetus, sich als Komponist beweisen zu müssen. Wie effektiv er dies beherrschte, wird in diesen und späteren Liedern deutlich.

Ebenfalls im besetzten Peking tätig war der Pianist Lao Zhicheng 老志誠 (19102006). 1943 vertonte Lao eine Reihe klassischer Gedichte, darunter Texte von Li Bai. ${ }^{19}$ Auch er wählte Gedanken in stiller Nacht, Einer Flöte lauschen in Luoyangs

18 Jiang Wenye: Jiang Wenye quanji, Band 5.2, Beijing: China Central Conservatory Press, 2016, S. $65-67,76 \mathrm{f}$. und $138-149$.

19 Lao Zhicheng: Lao Zhicheng yinyue zuopin xuanji shiyi, Changchun: Jilin Audio and Video Publishing House, 2002, S. 81 f., 87, 92-96 und 99. 
Frühlingsnacht und zusätzlich Jugendlied (Shaonian xing 少年行), Mond über dem Pass (Guan shang yue 關山月) und Krähen krächzen in der Nacht (Wu ye ti 鳥夜 啼). Die Textauswahl zeugt von der Beliebtheit bestimmter Gedichte Li Bais. Dennoch ist bemerkenswert, dass Lao nicht nur Abschieds- und Heimatgedichte wählte, sondern zwei, die aus der Perspektive der daheim gebliebenen Frau eines Kriegers geschrieben sind. Solche Texte werden häufig als Anklage an kriegstreibende Regime verstanden, doch können sie hier auch als Klage eines im besetzten Peking Zurückgebliebenen, um der eigenen Sicherheit willen Mundtoten gelesen werden.

Sowohl Jiang als auch Lao wurden zu Botschaftern der zwei aufeinanderfolgenden nordchinesischen Kollaborationsregime. ${ }^{20}$ Jiang Wenye komponierte zwischen 1938 und 1942 mehrere Hymnen und Propagandalieder und Lao Zhicheng wirkte als Pianist an Propagandaveranstaltungen mit. Die reine Anwesenheit und jegliche Aktivität solch prominenter Musiker wurde vonseiten der Regime genutzt, um kulturellen Wohlstand in den Gebieten unter japanischem Einfluss zu suggerieren. In Publikationen und Konzertprogrammen der `Kollaborateure ‘ finden sich Li-Bai-Vertonungen dreier prominenter Musiker. Kurz vor Ende des Krieges erschienen überraschend mehrere Li-Bai-Vertonungen in der wichtigsten Tageszeitung des Regimes. Noten in einer Tageszeitung waren ohnehin eine Seltenheit, doch nun, da Papier knapp war und Zeitungen in herabgesetzter Auflage gedruckt wurden, erscheinen sie gänzlich unerwartet. Im Mai 1944 wurde die Veröffentlichung eines Liederzyklus von Lao Zhicheng mit einer Abschrift der Melodie von Jugendlied angekündigt. ${ }^{21}$ Nur wenige Monate später findet sich in derselben Zeitung eine Vertonung von Gedanken in stiller Nacht, komponiert vom Geiger Li Yiming 李一 鳴, der ebenfalls regelmäßig für das Regime konzertierte. ${ }^{22}$

1944 gab Jiang Wenye in Peking mehrere Liederabende ${ }^{23}$ - unter anderem mit Li-Bai-Vertonungen - für die frisch Graduierten des Literaturinstitutes der staatlichen Peking-Universität. ${ }^{24}$ Mit Vertonungen von Johann Wolfgang

20 Für einen historischen Abriss zum Zweiten Sino-Japanischen Krieg siehe Susanne WeigelinSchwiedrzik: Chinesisch-Japanischer Krieg 1937-45, in: Das große China-Lexikon, hg. von Brunhild Staiger, Stefan Friedrich und Hans-Wilm Schütte unter Mitarbeit von Reinhard Emmerich; Darmstadt: Wissenschaftliche Buchgesellschaft, 2003, S. 137-139. Das japanisch besetzte Peking wurde von zwei aufeinander folgenden Klientenregimen regiert: ab Dezember 1937 von der Provisorischen Regierung der Republik China, ab März 1940 vom Politischen Rat von Nordchina, der nominell der in Nanjing ansässigen Neuorganisierten Regierung der Republik China (auch bekannt als Wang-Jingwei-Regime) untergeordnet war, aber weitgehend unabhängig agierte.

21 Lao Zhicheng: Shaonian xing, in: Huabei xinbao (01.05.1944), S. 7. Die Abschrift enthält nur die Melodie, für mehr war leider ’kein Platz .

22 Li Yiming: Jing ye si, in: Huabei xinbao (14.09.1944), S. 3.

23 [Anon.]: Zuoqu shengyue mingjia Jiang Wenye nianwu nianliu liang wan juxing geren duchang dahui, in: Shibao (25.02.1944), S. 4. Siehe auch Huabei xinbao (10.08.1944), S. 2.

24 [Anon.]: Jiang Wenye dingqi juxing zhong wai shici duchanghui, in: Xinminbao (29.03.1944), S. 2. 


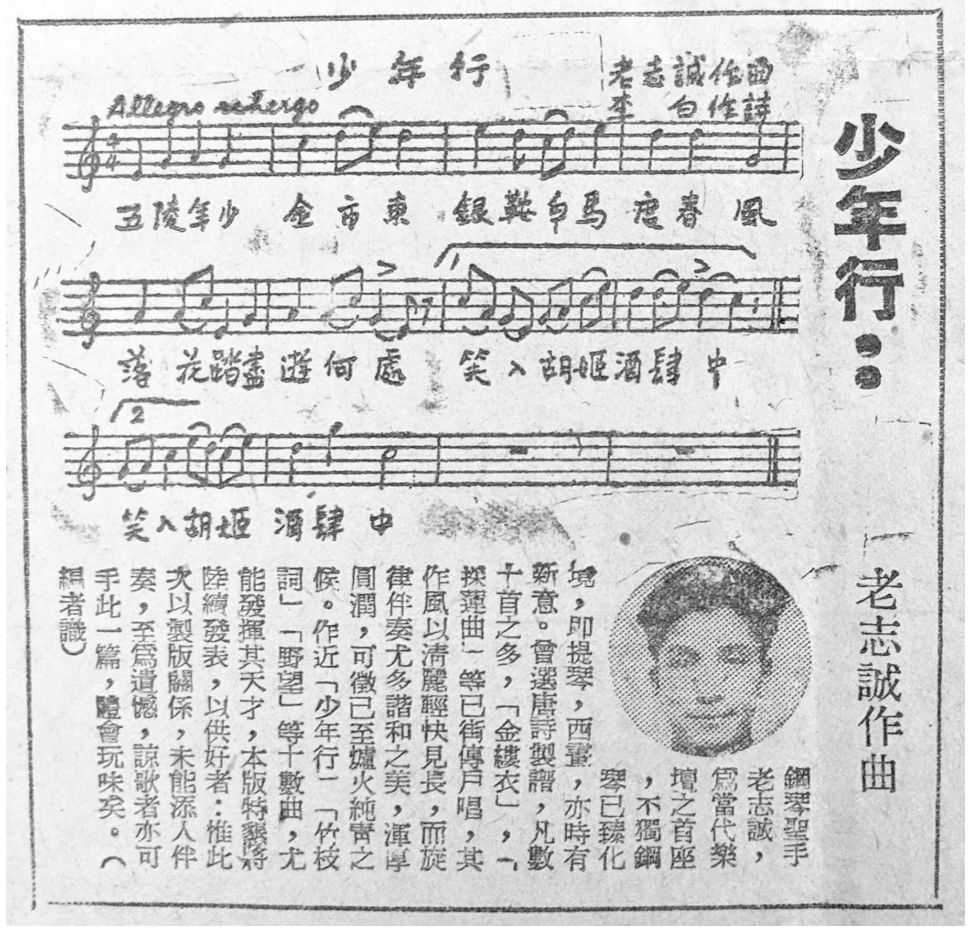

Abb. 3: Lao Zhicheng: Jugendlied, publiziert in der Huabei xinbao (01.05.1944)

von Goethe, Heinrich Heine, ${ }^{25}$ Tōson Shimazaki 島崎藤村 (1872-1943), Su Shi 蘇 軾 (1037-1101) und Li Bai war das Programm so zusammengestellt, dass es die Achsenmächte repräsentierte. (Das chinesische Kollaborationsregime hatte schon im Januar 1943 den Alliierten den Krieg erklärt.) Typisch ist dabei, dass Japan von einem zeitgenössischen Dichter repräsentiert wird, während China durch Li Bai und Su Shi zwar als Wiege 'pan-asiatischer Kultur, aber auch als entschieden >unmodern charakterisiert wird.

Die Verwendung von Li Bais Gedichten in solch hoch politisierten Kontexten bietet Anlass zu weiteren Überlegungen zum vielfachen Gebrauch und Missbrauch des identitäts- und traditionsstiftenden Charakters der Texte Li Bais. Li Bai kann als pan-asiatisch und unmodern das China-Bild der japanischen Besatzer bedienen, kann aber zugleich als Referenz auf die kulturelle Blütezeit Chinas als nationalistisch und subversiv gedeutet werden. Texte, die aufgrund ihrer Popularität die konkrete Assoziation mit Dichter und Entstehungskontext verlieren, ein Eigenleben unabhängig von den biografischen Details ihres Autors entwickeln und so zu diffus >chinesischem

25 Heinrich Heine mag in NS-Deutschland als Jude verfemt worden sein, in China war man jedoch wenig verständnis- und rücksichtsvoll, was die momentanen Limitationen im deutschen Selbstverständnis anging. So wurde zum Beispiel auch Felix Mendelssohn häufig gespielt und als Repräsentant Deutschlands verstanden. 
Volkseigentum ‘ werden, bieten sich zu solchem Ge- und Missbrauch an. So ließen sich auch die Texte Li Bais in allen Gebieten des kriegsgebeutelten China als obskure Referenz auf potenziell identitätsstiftende Wurzeln und als ein Anhaltspunkt auf dem Weg zu einer neuen staatlichen Konfiguration der Kulturnation einsetzen.

Die Menge an modernen Li-Bai-Vertonungen in China ist überraschend überschaubar. ${ }^{26} \mathrm{Li}$ Bai ist eindeutig reiner von Vielen . Das bezeugt auch der Umstand, dass die Vertonungen seiner Texte häufig in Zyklen `Tang-zeitlicher Gedichte zu finden sind - innerhalb derer Li Bai dann wiederum eine herausragende Rolle einnimmt. Doch auch Dichter wie Meng Haoran 孟浩然 (689/691-740), Bai Juyi 白居易 (772846), Zhang Ji 張繼 (715-779) und Su Shi erfreuen sich bei chinesischen Komponist*innen großer Beliebtheit. Daneben wurden in der ersten Hälfte des zwanzigsten Jahrhunderts Texte moderner Dichter wie Guo Moruo 郭沫若 (1892-1978) und Xu Zhimo 徐志摩 (1897-1931) von ihren musikalischen Zeitgenossen gerne vertont.

Als symptomatisch für Li Bais erhöhte Popularität außerhalb von China mag gelten, dass gerade Chinesinnen im Ausland häufig auf Texte von ihm zurückgreifen. Li-Bai-Vertonungen finden sich zum Beispiel in den Werken chinesischer Auslandsstudenten auf der Suche nach chinesischen Stilelementen, wie Doming Lam (Lin Yuepei 林樂培, ${ }^{\star} 1926$ ), gerade aus Kanada in Kalifornien angekommen, im Vorwort zu seinen 1960 vollendeten drei Nachtliedern nach Texten Li Bais angibt. Die drei Lieder On a Quiet Night, The Ballad of an Autumn Night und Three with the Moon and his Shadow sind auf den chinesischen Text gesetzt. ${ }^{27}$ Direkt nach seiner Rückkehr nach Hong Kong komponiert er 1964 Two Farewell Songs from the Poems of Li-Po für hohe Stimme und Flöte - dieses Mal in englischer Übersetzung. ${ }^{28}$ Lams Textauswahl ist bezeichnend: Die drei Nachtlieder thematisieren die Einsamkeit des Reisenden, Saying Farewell to a Friend ist einem amerikanischen Kommilitonen gewidmet und Leaving White Emperor City at Dawn einem chinesischen Kommilitonen als Aufruf zur baldigen Rückkehr in seine Heimat. Auch Yang Liqing 楊立青 (1942-2013), gerade in Hannover angekommen, vertonte zwischen 1981 und 1982 vier Gedichte aus der Tang-Dynastie für Frauenstimme, Schlagzeug und Klavier. ${ }^{29}$ Neben Abschied von Li Bai sind Texte von Bai Juyi, Meng Haoran und Li Jiayou 李 嘉祐 in dem Zyklus enthalten.

Eine Recherche in den Beständen der Bibliothek des Pekinger Zentralkonservatoriums ergab weniger als einhundert Kunstlieder und Arrangements für Chor. Für listenartige Ausführungen zu chinesischen Li-Bai-Vertonungen siehe Jin Honglian/Ma Yufeng: Jiyu Li Bai shici chuangzuo de yishu gequ gaishu yu yanjiu, in: Yinyue Chuangzuo 10 (2014), S. 153-155; Yang: Li Bai shici gequ yanjiu.

27 Doming Lam: Li Bai yeshi san shou, in: Mei yue xin ge Nr. 146, 149, 152, Taipei: Yuen-Yuhn Music Publishing Company, 1963.

28 Doming Lam: Two Farewell Songs from the Poems of Li-Po (in English). Duo for High Voice and Flute op. 13, Taipei: Yuen-Yuhn Music Publishing Company, 1964.

29 Yang Liqing: Vier Gedichten aus [sic!] Tang-Dynastie für Frauenstimme, Schlagzeug und Klavier, Shanghai: Shanghai Conservatory Music Press, 1981-1982. Eine Analyse der Lieder findet sich in Tang Rong: Yang Liqing »Tang shi si shou« yinyue fenxi, in: Music Research 6 (2018), S. $26-42$. 
Der wohl berühmteste Zyklus chinesischer Li-Bai-Vertonungen nach 1949 stammt aus der Feder Ma Sicongs 馬思聰 (1912-1987); seine Sechs Gedichte Li Bais (Li Bai shi liu shou 李白詩六首) entstanden in den frühen 1960er Jahren. ${ }^{30}$ Der Zyklus basiert auf sechs weiteren sehr beliebten Gedichten Li Bais: Sehnen in Chan'gan (Chang xiang si 長相思), Lasst uns trinken (Jiang jin jiu 將進酒), Der Weg ist schwer I und II (Xing lu nan 行路難), Mond über dem Pass und Abschied von Jingmen (Du Jingmen songbie 渡荊門送別). In den 1970er Jahren nahm sich Qu Wenzhong 屈文 中 (1942-1992) der Texte an. Sein Liederzyklus zu vier Gedichten Li Bais (Li Bai shi zuge si shou 李白詩組歌四首) von 1976 enthält zwei Lieder für Stimme und zwei Arrangements für gemischten Chor, jeweils mit Klavierbegleitung. ${ }^{31}$

Li Bai erfreut sich, gerade im Vergleich mit anderen vormodernen Autoren und Texten, steter Beliebtheit bei chinesischen Komponist*innen. Auch seine Person ist wiederholt zum Gegenstand musikdramatischer Annäherungen geworden. Zwei biografische Großprojekte der letzten Jahre sollen daher nicht unerwähnt bleiben: die Oper Poet Li Bai (Shiren Li Bai 詩人李白) von Guo Wenjing 郭文景 (*1956), uraufgeführt 2007 in Colorado, ${ }^{32}$ und die Musik zum Ballett Li Bai von Zhang Qu 張渠, das vom China National Opera \& Dance Drama Theater in Auftrag gegeben und 2018 in Chengdu uraufgeführt wurde. ${ }^{33}$ Beide Projekte bezeugen Li Bais ungebrochenen Status als Kulturikone, aber auch das Interesse an neuen Zugängen zu seinem Schaffen.

\section{Literatur}

[Anon.]: Jiang Wenye dingqi juxing zhong wai shici duchanghui 江文也定期舉行中外詩詞 獨唱會 [Jiang Wenye gibt bald einen Liederabend mit chinesischen und ausländischen Gedichten], in: Xinminbao 新民報 (29.03.1944), S. 2.

[Anon.]: Zuoqu shengyue mingjia Jiang Wenye nianwu nianliu liang wan juxing geren duchang dahui 作曲聲樂名家江文也廿五廿六兩晚舉行獨唱大會 [Der Komponist und Sänger Jiang Wenye gibt an den Abenden des 25. und 26. große Liederabende], in: Shibao 實 報 (25.02.1944), S. 4 .

Asian Performing Arts Council: Poet Li Bai, www.asianperformingartscouncil.org/poet-li-bai (28.02.2021).

China National Opera \& Dance Drama Theatre: Minzu Wuju »Li Bai«民族舞剧《李白》 [Ethnisches Ballett »Li Bai«]. www.cnoddt.com/cont.aspx?newsId=3920\&TId=10 (28.02.2021).

30 Ma Sicong: Li Bai shi (liu shou), in: Mei yue xin ge Nr. 154, 159, 165, 170, 175, Taipei: Yuen-Yuhn Music Publishing Company, 1963-1964. Eine Analyse der Lieder findet sich in: Peng: Juan wei wang yue kong chang tan.

31 Gao Hongbo: Qu Wenzhong yinyue zuopin yanjiu, www.sin80.com/pub/wut-man-chungc7c96c04 (28.02.2021).

32 Asian Performing Arts Council: Poet Li Bai, www.asianperformingartscouncil.org/poet-li-bai (28.02.2021).

33 China National Opera \& Dance Drama Theatre: Minzu Wuju »Li Bai«, www.cnoddt.com/cont. aspx?newsId=3920\&TId=10 (28.02.2021). 
Falkenhausen, Lothar von: Suspended Music. Chime-Bells in the Culture of Bronze Age China, Berkeley: University of California Press, 1993.

FitzGerald, Carolyn: Fragmenting Modernisms. Chinese Wartime Literature, Art, and Film, 1937-49, Leiden/Boston: Brill, 2013, https://doi.org/10.1163/9789004250994.

Fu, Poshek: Passivity, Resistance, and Collaboration. Intellectual Choices in Occupied Shanghai, 1937-1945, Stanford, CA: Stanford University Press, 1993.

Fu, Poshek: The Ambiguity of Entertainment. Chinese Cinema in Japanese-Occupied Shanghai, 1941 to 1945, in: Cinema Journal 37/1 (1997), S. 66-84, https://doi.org/10.2307/1225690.

Gao Hongbo 高洪波: Qu Wenzhong yinyue zuopin yanjiu 屈文中声乐作品研究 [Forschungen zu den Kompositionen von Qu Wenzhong], www.sin80.com/pub/wut-man-chungc7c96c04 (28.02.2021)

Gunn, Edward: Unwelcome Muse. Chinese Literature in Shanghai and Peking, 1937-1945, New York: Columbia University Press, 1980, https://doi.org/10.7312/gunn94514.

Howard, Joshua. »Music for a National Defense«. Making Martial Music during the Anti-Japanese War, in: Cross-Currents 4/1 (2015), S. 238-284, https://doi.org/10.1353/ach.2015.0016.

Jiang Wenye 江文也: Jiang Wenye quanji 江文也全集 [Jiang Wenye Gesamtausgabe], Band 5.2, Beijing: China Central Conservatory Press, 2016.

Jin Honglian 金红莲/Ma Yufeng 马玉峰: Jiyu Li Bai shici chuangzuo de yishu gequ gaishu yu yanjiu 基于李白诗词创作的艺术歌曲概述与研究 [Untersuchungen zu Li-Bai-Vertonungen], in: Yinyue Chuangzuo 音乐创作 10 (2014), S. 153-155.

Kroll, Paul W.: Poetry of the T'ang Dynasty, in: The Columbia History of Chinese Literature, hg. von Victor H. Mair, New York: Columbia University Press, 2001, S. 274-313.

Lam, Doming 林樂培: Li Bai yeshi san shou 李白夜詩三首 [Drei Nachtlieder von Li Bai], in: Mei yue xinge 每月新歌 [Jeden Monat neue Lieder] Nr. 146, 149, 152, Taipei: Yuen-Yuhn Music Publishing Company 樂韻出版社, 1963.

Lam, Doming 林樂培: Two Farewell Songs from the Poems of Li-Po (in English). Duo for High Voice and Flute op. 13, Taipei: Yuen-Yuhn Music Publishing Company 樂韻出版社, 1964.

Lao Zhicheng 老志诚: Lao Zhicheng yinyue zuopin xuanji shiyi 老志诚音乐作品选集拾 遗 [Ausgewählte Kompositionen von Lao Zhicheng], Changchun: Jilin Audio and Video Publishing House, 2002.

Lao Zhicheng 老志誠: Shaonian xing 少年行 [Jugendlied], in: Huabei xinbao 華北新報 (01.05.1944), S. 7.

Li Yiming 李一鳴: Jing ye si 靜夜思 [Stille Nachtgedanken], in: Huabei xinbao 華北新報 (14.09.1944), S. 3.

Lindorff, Joyce: Missionaries, Keyboards and Musical Exchange in the Ming and Qing Courts, in: Early Music 32/3 (2004), S. 403-414, https://doi.org/10.1093/em/32.3.403.

Liu Ching-Chih: A Critical History of New Music in China, übers. von Caroline Mason, Hong Kong: Chinese University Press, 2010.

Liu Qinqin 刘沁沁: Shangbie chu, yin jianjue - Xian Xinghai yishu gequ »Yi Qin'e» fenxi 伤别处, 音塵决——冼星海艺术歌曲《忆秦娥》分析 [Ort der schmerzlichen Trennung, Nachrichten brechen ab. Analyse von Xian Xinghais Kunstlied »Erinnerung an die Schönheit von Qin«], in: Journal of Xinghai Conservatory of Music 3 (2011), S. 80-85. 
Ma Sicong 馬思聰: Li Bai shi (liu shou) 李白詩 (六首) [Li Bai Gedichte (Sechs Stücke)], in: Mei yue xin ge 每月新歌 [Jeden Monat neue Lieder] Nr. 154, 159, 165, 170, 175, Taipei: Yuen-Yuhn Music Publishing Company 樂韻出版社, 1963-1964.

Mittler, Barbara: Dangerous Tunes. The Politics of Chinese Music in Hong Kong, Taiwan, and the People's Republic of China since 1949, Wiesbaden: Harrassowitz, 1997.

Peng Li 彭丽: Juan wei wang yue kong chang tan. Xi Ma Sicong de yishu gequ »Li Bai shi liu shou 《卷帷望月空长叹. 析马思㙂的艺术歌曲《李白诗六首》[Ich rolle den Vorhang zurück, blicke in den Mond und seufze tief. Analyse von Ma Sicongs Kunstliedern »Sechs Gedichte Li Bais«], in: Lu Xun Yiyuan魯迅藝苑 4 (2006), S. 57-62.

Tang Rong 唐荣: Yang Liqing »Tang shi si shou« yinyue fenxi 杨立青《唐诗四首》音乐分 析 [Musikalische Analyse von Yang Liqings »Vier Tang-Gedichte«], in: Music Research 6 (2018), S. 26-42.

Tang Xiaobing: Radio, Sound Cinema, and Community Singing. The Making of a New Sonic Culture in Modern China, in: Twentieth-Century China 45/1 (2020), S. 3-24, https://doi. org/10.1353/tcc.2020.0005.

Wang, David Der-Wei: The Lyrical in Epic Time. The Music and Poetry of Jiang Wenye, in: The Lyrical in Epic Time. Modern Chinese Intellectuals and Artists Through the 1949 Crisis, New York: Columbia University Press, 2015, S. 193-235, https://doi.org/10.7312/ wang17046-008.

Weigelin-Schwiedrzik, Susanne: Chinesisch-Japanischer Krieg 1937-45, in: Das große ChinaLexikon, hg. von Brunhild Staiger, Stefan Friedrich und Hans-Wilm Schütte unter Mitarbeit von Reinhard Emmerich, Darmstadt: Wissenschaftliche Buchgesellschaft, 2003, S. 137-139.

Xian Xinghai 洗星海: Xian Xinghai quanji 洗星海全集 [Xian Xinghai Gesamtausgabe], hg von Xian Xinghai quanji bian weiyuanhui 洗星海全集编委员会 [Xian Xinghai-Gesamtausgaben-Komitee], Band 2, Guangzhou: Guangdong Higher Education Press, 1990, S. 328-331.

Yang Liqing 楊立青: Vier Gedichten aus [sic!] Tang-Dynastie für Frauenstimme, Schlagzeug und Klavier, Shanghai: Shanghai Conservatory Music Press, 1981-1982.

Yang Sihui 杨思慧: Li Bai shici gequ yanjiu 李白诗词歌曲研究 [Untersuchungen zu Vertonungen der Gedichte Li Bais], in: Journal of Anhui University of Technology 32/6 (2015), S. 59-62.

L. Odila Schröder hat Chinesisch, Politikwissenschaft und Wissenschaftsgeschichte in Peking, Cambridge und Heidelberg studiert. Ihren Master in Sinologie und Transcultural Studies schloss sie in Heidelberg mit einer Arbeit über fonografische Dokumentationen von Pekinger Alltagsgeräuschen ab. Seit 2017 promoviert sie im Rahmen des vom ERC geförderten Projekts »Cultures of Occupation in Twentieth-Century Asia « an der University of Nottingham zu Musik und auditiven Propagandastrategien chinesischer Kollaborationsregime im japanisch besetzen Peking (1937-1945). 


\title{
Der doppelte Po und die Musik
}

Rätoromanisch-chinesische Studien, besonders zu

Li Po, Harry Partch und Chasper Po

\author{
Herausgegeben von \\ Mathias Gredig, Marc Winter, \\ Rico Valär und Roman Brotbeck \\ Redaktionelle Mitarbeit \\ Daniel Allenbach
}

Königshausen \& Neumann 
Bibliografische Information der Deutschen Nationalbibliothek

Die Deutsche Nationalbibliothek verzeichnet diese Publikation in der Deutschen

Nationalbibliografie; detaillierte bibliografische Daten sind im Internet über http://dnb.d-nb.de abrufbar.

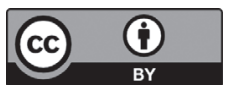

Dieses Werk ist lizenziert unter der Creative Commons Attribution 4.0 Lizenz (BY). Diese Lizenz erlaubt unter Voraussetzung der Namensnennung des Urhebers die Bearbeitung, Vervielfältigung und Verbreitung des Materials in jedem Format oder Medium für beliebige Zwecke, auch kommerziell. (Lizenztext: https://creativecommons.org/ licenses/by/4.0/deed.de) Die Bedingungen der Creative-Commons-Lizenz gelten nur für Originalmaterial. Die Wiederverwendung von Material aus anderen Quellen (gekennzeichnet mit Quellenangabe) wie z. B. Schaubilder, Abbildungen, Fotos und Textauszüge erfordert ggf. weitere Nutzungsgenehmigungen durch den jeweiligen Rechteinhaber.

Erschienen 2021 im Verlag Königshausen \& Neumann GmbH

(C) bei den Autoren

Die Druckvorstufe dieser Publikation wurde vom Schweizerischen Nationalfonds zur Förderung der wissenschaftlichen Forschung unterstützt.

\section{FNSNF}

SCHWEIZERISCHER NATIONALFONDS

ZUR FÖRDERUNG DER WISSENSCHAFTLICHEN FORSCHUNG

Wir danken der Kulturförderung des Kantons Graubünden.

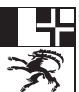

Kulturförderung Graubünden. Amt für Kultur

Promoziun da la cultura dal Grischun. Uffizi da cultura

Promozione della cultura dei Grigioni. Ufficio della cultura

SWIISSLOS

Hochschule der Künste Bern

www.hkb.bfh.ch

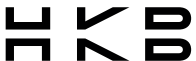

Hochschule der Künste Bern

Haute école des arts de Bern

Bern University of the Arts

Umschlag: skh-softics / coverart

Umschlagabbildung: Lea Gredig

Print-ISBN 978-3-8260-7180-5

PDF-ISBN 978-3-8260-7233-8

DOI $10.26045 /$ po

https.//doi.org/10.36202/9783826072338

Gedruckt auf säurefreiem, alterungsbeständigem Papier

Printed in Germany

www.koenigshausen-neumann.de

www.ebook.de

www.buchhandel.de

www.buchkatalog.de 


\section{Inhalt}

Prolog

Dumenic Andry

Chasper Pos Humor

Renzo Caduff

Chasper Pos rhythmische Versgestaltung - eine shinkende Mähre`?

Rico Valär

Rätoromanische Nachdichtungen chinesischer Lyrik bei

Gian Fadri Caderas und Peider Lansel

Eine Spurensuche

Mathias Gredig

China in rätoromanischen Zeitungen, Zeitschriften

und literarischen Texten

Marion Eggert

Schwalbenflug in Gedichten von Li Bai und Chasper Po

Thomas Geissmann

Die Rolle der Gibbons beim chinesischen Dichter Li Bai

Marc Winter

„Chinas Dichterfürst«

Die Rezeption Li Bais als literarischer Superstar im Westen

Eva Schestag

"A most difficult man«

Ezra Pound als Übersetzer von Li Bai, mit einem Seitenblick auf Shigeyoshi Obata

Odila Schröder

Chinesische Li-Bai-Vertonungen in Jahren der Unruhe 
Mathias Gredig

Quantitative Überlegungen zum Phänomen

der Li-Bai-Vertonungen im Westen

Mit Beobachtungen zu drei Vertonungen des Gedichtes

Chun ye Luo cheng wen di (In einer Frühlingsnacht in Luoyang eine Flöte hören)

\section{Gesine Schröder}

"Die Hüften schwingen sich nun nicht mehr»

Li-Bai-Vertonungen von Komponistinnen

Heinrich Aerni

Li-Bai-Vertonungen in der Schweiz

Matthias Schmidt

Übersetzung ohne Original?

Gustav Mahler, Anton Webern und Li Bai

\section{Christoph Haffter}

Szenen der Selbstenttäuschung

Hanns Eislers Die rote und die weiße Rose nach Li Bai und die Antinomien der Kriegslyrik

Thomas Meyer

„Wunderlich im Spiegelbilde»

Zu einigen Vertonungen des Pavillon-Gedichts

\section{Mathias Gredig}

Gedanken über Li Bais Jing ye si (Gedanken in einer stillen Nacht)

und dessen Vertonungen im Westen

\section{Martin Skamletz}

"I've turned into a great reviser."

Lee Hoibys Vertonung von Li Bais The River-Merchant's Wife: A Letter

und ihr Bezug zu Harry Partch

\section{Martin Skamletz}

"Of course I am a weak shadow of Lee Hoiby as a Kitharist."

Five letters by Harry Partch, 1948-1958

\section{Marc Kilchenmann}

Ben Johnstons Verhältnis zu Harry Partch und seine Three Chinese Lyrics 
Eleni Ralli

Parallelen und Modifikationen der Notation in verschiedenen Quellen

von Harry Partchs Seventeen Lyrics by Li Po

Schwierigkeiten und Transkriptionsvorschläge

Charles Corey

Gesture and Intention in the Art Songs of Harry Partch

Caspar Johannes Walter

Sprechmelodie als Quelle von Melodik und Harmonik

The Intruder aus Harry Partchs Li-Bai-Vertonungen

Roman Brotbeck

Der Sprechgesang bei Arnold Schönberg und Harry Partch

Eine Annäherung

Namensregister 